\title{
A ONDA P E AS HIPERTROFIAS AURICULARES (1)
}

\author{
Acad. RAPHAEL GIANNELLA \\ Interno voluntárin da 2.a M. H. da Santa Casa, Serviço do Prof. Rubião \\ Meira (3.a Cadeira de Clínica Médica da Faculdade de Medicina da Uni- \\ versidade de São Paulo)
}

\section{JUSTIFICATIVA}

Foi após o aperfeiçoamento da aparelhagem eletrocardiográfica que se começou a estudar as deflexões auriculares. De fato, enquanto as anomalias do complexo ventricular, nos últimos 15 anos, foram pesquizadas, os nossos conhecimentos sobre a aurícula humana e sua patologia, eram, até bem pouco tempo, duvidosos. Isto é explicado pelo fato de que com os aparelhos antigos nem sempre conseguiamos uma onda $P$ nítida, o que se realiza, atualmente, com os eletrocardiógrafos modernos.

Em vitrude dos trabalhos nacionais se referirem, apenas, de passagem e somente, sobre determinados aspectos, foi que, por idéiả e orientação de nosso mestre, dr. Luiz V. DÉcourt, nos propuzemos a encarar mais de perto o problema da ONDA P E AS HIPERTROFIAS AURICULARES.

Para comodidade de exposição dividiremos nosso trabalho em 3 partes fundamentais:

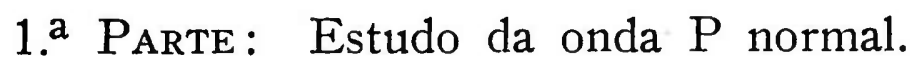

2. a Parte: Estudo da onda $\mathrm{P}$ e as hipertrofias auriculares.

3. Parte: Exposição de nossos dados e conclusões.

\section{1. ${ }^{\mathrm{a}}$ Parte}

\section{ESTUDO DA ONDA P NORMÅL}

\section{RECONHECIMENTO E SIGNIFICAÇÃO}

A onda $\mathrm{P}$, primeiro acidente positivo que aparece no registro elétrico do cíclo cardíaco, precede, por um determinado intervalo, o, facilmente reconhecivel complexo ventricular: QRS.

(1) Trabalho apresentado no $2 .^{\circ}$ Congresso Médico-Estudantino organizado pelo Departamento Cientifico do Centro Acadêmico "Oswaldo Cruz". em 10 de outubro de 1941 (Sessão de Clínica Médica e Clínica Cirurgica). 
Representa ește acidente, a excitação da musculatura auricular, daí a designação de "complexo auricular". Entretanto, convem assinalar que o seu registro somente se efetua quando a onda excitadora já. se propagou por toda a musculatura auricular.

Precede a onda $\mathrm{P}$, de $0^{\prime \prime}, 02$, a contração auricular.

\section{NOMENCLATURA}

A designação de onda $P$, com aliás a de todas as deflexões eletrocardiográficas, foi proposta por EINTHOVEN, propositadamente, sem significação alguma, para que com os trabalhos posteriores não se criassem confusões sobre a sua significação.

A nomenclatura proposta por Kraus e NicolaI (citado por ToLEDo), onde o $\mathrm{P}$ é designado por $\mathrm{A}$ (de aurícula) não apresenta nenhuma vantagem prática.

\section{ALTURA OU AMPLITUDE}

A onda $\mathrm{P}$ normal apresenta uma altura ou amplitude que oscila entre $0^{\mathrm{mm}}, 7$ a $2^{\mathrm{mm}}, 5$ na derivação onde esta altura é máxima.

De acordo com Shipley e Hallaran (citados por PardeE), a maior amplitude de $\mathrm{P}$ é em DII, em cerca de $90 \%$ dos casos.

Para Ashmann e Hull (1) a maior altura de $\mathrm{P}$ é vista em DII e a menor em DI, particularmente, em indivíduos hipostênicos.

A maior amplitude em DII indica que o eixo elétrico das aurículasé, aproximadamente, paralelo a esta derivação, situando-se ao redor de $+60 .^{\circ}$.

A altura de $\mathrm{P}$ é ,muitas vezes dificil de ser determinada com exatidão, apresentando variações de ciclo para ciclo e, especialmente, com os movimentos respiratórios na arritmia sinusal.

Para Ashman e Hull (1), a amplitude normal da onda $\mathrm{P}$, tomando a medida de 100 indivíduos normais, é para DI igual o $0^{\mathrm{mm}}, 55$, para DII igual a $1^{\mathrm{mm}}, 364$, para DIII igual a $0^{\mathrm{mm}}, 83$.

\section{DURAÇÃO OU LARGURA}

Pardee (4), em 100 estudantes normais, numa série de 26 indivíduos, encontrou uma duração variavel entre $0^{\prime \prime}, 08$ a $0^{\prime \prime}, 10$. Num único caso a duração foi de $0^{\prime \prime}, 06$.

Shipley e Hallaran (citados por Pardee), encontraram, em 3 registros de 200 indivíduos normais, uma duração igual a $0^{\prime \prime}, 12$; em 13 registros acharam-na igual a $0^{\prime \prime}, 11$.

Ashman e Hull (1), encontraram variações entre $0^{\prime \prime}, 06$ a $0^{\prime \prime}, 11$ e, numa única-circunstância, acharam, em 100 indivíduos examinados, uma duração excendendo $0^{\prime \prime}, 10$, daí tomarem $0^{\prime \prime}, 11$ como limite máximo da normalidade. 
E preciso considerar que a derivação na qual a duração da onda $P$ é maior é, frequentemente, a segunda. Não conhecemos o fator que torna este tempo desigualmente curto, mas, em geral, é encontrado nos corações muito rápidos e em crianças.

$\mathrm{Na}$ criança a duração de $\mathrm{P}$ varia de $0^{\prime \prime}, 04$ até $0^{\prime \prime}, 08$, com u'a media agual a $0^{\prime \prime}, 06$ (PARDEE - 4).

\section{DIREÇÃO}

A onda ${ }^{P}$ é sempre positiva em $\mathrm{DI}$ e, raramente, invertida em DIII, cerca de $4 \%$ dos casos; ou difásica, em cerca de $2 \%$ dos casos.

\section{FORMA}

A onda $\mathrm{P}$ é representada no eletrocardiograma por uma elévação arredondada, positiva, isto é, acima da linha de iso-eletricidade. Outras vezes, vê-se um achatamento apical e, em outras eventualidades, a existência de incisuras localizadas no apice. As incisuras apicais, por muito tempo, foram tidas como patológicas; entretanto, pesquizas de LEwis e Gildes (citados por RAmos) vieram demonstrar a presença de incisuras apicais em indivíduos jovens, sem transtornos cardíacos.

Ligeiros entalhes de $\mathrm{P}$ foram encontrados numa ou mais derivações, em cerca de $32 \%$ dos casos estudados por Ash man e Hull (1). $\mathrm{O}$ entalhe era frequentemente evidente em DII e se colocava muito próximo ao ápice do complexo auricular.

\section{CONCLUSÃO}

Quando encontrarmos uma onda $\mathrm{P}$ onde todos estes itens: altura, duração, direção e forma, são normais, podemos concluir que a contração auricular decorre segundo um percurso normal e através de u'a musculatura tambem normal.

\section{2..$^{\text {PARTE }}$}

\section{O ESTUDO DA ONDA P E AS HIPERTROFIAS AURICULARES}

Antes de estudarmos as alteraçōes que se produzem na onda $P$ quando dos aumentos auriculares, vejamos, em poucas palavras, em que casos essas hipertrofias aparecem.

Como resultado da hipertrofia de uma ou de outra câmara cardíaca, produzem-se as, muito frequentemente, observadas alterações eletrocardiográficas. A hipertrofia muscular nem sempre é o resultado de uma lesão miocárdica evidente,pois, pode proceder de causas 
puramente mecânicas, que demandem um aumento da propulsão do sangue pela câmara afetada.

Cuidaremos, no presente estudo, das hipertrofias auriculares consequentes à lesões das valvas mitrais e das hipertrofias consequentes à sobrecarga do ventrículo direito, por hipertensão na pequena circulação.

Assim, na estenose mitral pura, encontramos, nos casos avançados, ao lado de uma atrofia muscular do ventriculo esquerdo, com acentuada diminuição de seu volume, uma dilatação da aurícula esquerda e uma saliência muito apreciavel da via de defluvio do ventriculo direito.

A musculatura relativamente delgada da aurícula esquerda, incapaz de uma hipertrofia consideravel, distende-se mais ou menos rapidamente. Com a distensão e, consequentemente, com as deficiências da nutrição, aparecem perturbações para o lado da excitabilidade e da estimulogenese auricular.

$\mathrm{Na}$ insuficiência mitral, encontramos uma hipertrofia predominante do ventriculo esquerdo com dilatação da aurícula homônima.

Com isso, compreendemos, facilmente, que estas duas lesões mitrais produzem alterações, até certo ponto, opostas para o lado do ventrículo preponderante. Entretanto, a coexistência destes dois processos é a regra, portanto, os aspectos eletrocardiográficos dependem da predominância de uma lesão ou de outra.

\section{ALTERAÇÕES ELETROCARDIOGRÁFICAS DÁ ONDA P NA ESTENOSE MITRAL: P mitral}

Winternitz (9) descreve, na estenose mitral, alteraçốes na onda $\mathrm{P}$ que julga muito características da moléstia. $A$ onda $P$ ẹm $D I$ e DII apresenta-se entalhada, bifida ou dupla. A amplitude vai de $1^{\mathrm{mm}}, 5$ até $2^{\mathrm{mm}}, 5$. Em DIII é difásica ou negativa. Sua duração ultrapassa nas três derivações $0^{\mathrm{mm}}, 10$. O aumento desta duração aparece, em geral, nas complicações, por exemplo, com insuficiência mitral, lesãõ das válvulas aórticas, insuficiência cardíaca e moléstias agudas ou crônicas.

Berliner e Master (2), em 69 casos de estenose mitral pura, com ritmo regular, encontraram em 61 , a onda $P$ entalhada. Em 16 casos de estenose mitral pura, com ritmo sinusal regular, encontraram a onda $\mathrm{P}$ entalhada em uma ou mais derivações, e em 6 desses casos havia, além disso, alargamento de $\mathrm{P}$, em, pelo menos, uma derivação. A média da altura em DII, nesses 16 casos, era de $1^{\mathrm{mm}}, 63$; em, somente, 4 dos 16 casos era mais alta de $2^{\mathrm{mm}}$ e, em 4 outros casos, era de $2^{\mathrm{mm}}$.. $\mathrm{O}$ aumento, por conseguinte, na amplitude de $\mathrm{P}$ era pouço frequente nos casos de estenose mitral pura.

Em metade dos casos de doença mitral pura, havia hipertrofia das aurículas direita e esquerda. Em todos estes casos havia uma onda $\mathrm{P}$ alta, de $2^{\mathrm{mm}}$ a $3^{\mathrm{mm}}, 5$ ou fibrilação auricular. Nos casos onde somente a aurícula esquerda estava hipertrofiada a onda $\mathrm{P}$ era de 
altura normal e em nenhum caso havia fibrilação auricular. Poder-seia assinalar então que um apreciavel aumento da onda P, com entalhes, seria patognomônico da dilatação de ambas as auriculas.

A mais alta onda $\mathrm{P}$ ( $\left(3^{\mathrm{mm}}, 5\right.$ ou mais $)$ frequentemente entalhada, foi encontrada nos casos de associação de estenose mitral com estenose tricuspide.

No grupo dos casos estudados por BerLiner e Master (2), onde a estenose mitral se associava com a insuficiência aórtica, a média de altura de $\mathrm{P}$ não era maior do que a da estenose mitral pura. A altura de $\mathrm{P}$ não excedeu a $2^{\mathrm{mm}}, 5$ enquanto, foram encontradas, em todos os casos, algumas Incisuras.

Raramente encontraram o alargamento da onda $\mathrm{P}$ na insuficiencia mitral e as incisuras só foram observadas em metade dos casos.

Schlomka e Klein (6), estudaram, tambem, as alterações da onda $\mathrm{P}$ não somente nos processos mitrais como nos casos de coração pulmonar ("Cor pulmonale") estudos estes posteriores aos de WinTERNITZ e que foram publicados em 1938

Estes dois autores descreveram a onda $\mathrm{P}$ - tipo mitral, como constituida por um P3 relativamente chato, enquanto que, com o aumento progressivo do desvio do eixo elétrico para a direita, aumentavam $\mathrm{P} 1$ e $\mathrm{P} 2$.

Em resumo, de acordo com os trabalhos vistos, em casos de hipertrofia da aurícula esquerda, ou não encontramos alterações da onda auricular (BerLiner e MASTER) ou estas se revelam por um $P 1$ e P2 entalhados, bifidos e um P3 chato ou difásico (Winternitz, Schlomka e Klein).

\section{SEGUNDO TIPO DE ALTERAÇOES DA ONDA P - P pulmonar}

Ao lado dessas alterações de $\mathrm{P}$, encontramos, ainda, um segundo tipo, que se distingue nitidamente, do $\mathrm{P}$ mitral e que pertence a um grupo unitário de moléstias.

Em Winternitz (9) encontramos: "Esta anomalia é um característico geral dos casos nos quais encontramos uma sobrecarga na pequena circulação com aumento da artéria pulmonar e hipertrofia. consecutiva do coração direito".

Classicamente, esta anomalia já tinha sido descrita no enfisema pulmonar essencial (juvenil) que é seguido por uma insuficiência cardíaca. Winternitz encontrou-a, porem, nos casós de alterações cardíacas da cifo-escoliose, nos casos de insuficência cardíaca por tuberculose pulmonar fibrosa, em processos pleurais, assim como no enfisema do velho, em bronquiectasia crônica e em 3 casos de estenose pulmonar congênita.

Winternitz (9) descreve o "P pulmonar" com os seguintes caractéres: é normal em DI, ds vezes, diminuido ou mesmo isoelétrico. Em DII e DIII apresenta-se aumentado. Aumentos na altura, de 3 a $4^{\mathrm{mms}}$. não são raros. Nos casos puros, não há alargamento de $\mathrm{P}$ alem de $0^{\prime \prime}, 10$. Eventualmente, pode aparecer um pequeno entalhe, 
porem, tal fato, não pertence ao quadro típico. $\mathrm{O}$ aumento de $\mathrm{P}$ é naais acentuado em DII e o intervalo PQ é normal em todos os casos.

De um modo geral, o $P$ pulmonar torna-se mais evidente na inspiração, pois, na expiração há maior elevação de P1 e achatamento de P2 e P3, tornando o quadro menos evidente. Estas alterações respiratórias de $\mathrm{P}$ aparecem, livremente, nos casos de excursñes respiratórias grande, com leslocabilidade cardíaca consequente.

Certos estados podem trazer uma alteração do $\mathrm{P}$ pulmonar, temporária ou permanente, assim, nos danos cardíacos com diminuição do potencial ventricular, pode haver um achatamento da onda $P$ que faz desaparecer o quadro típico. Winternitz (9) observou, por duas vezes, este fato, nos estados de envenenamento pela digital e estrofantina, podendo a onda $\mathrm{P}$ tornar-se até negativa.

Segundo observações do mesmo autor, nunca foi verificado o aparecimento de filtração auricular nos casos de $\mathrm{P}$ pulmonar, como se dá, contrariamente, nos casos de $\mathrm{P}$ mitral, ou pela evolução do processo ou pela ação da digital.

Schlom Ka e Klein (6) descreveram o P pulmonar com os mes. mos caracteres. Assim, descreveram, no pulmonares (por exemplo, nos enfisematosos), um tipo com desvio do eixo elétrico para a direita, onde, de um lado, P3 e principalmente $\mathrm{P} 2$ eram, muito aumentados em relação a $\mathrm{P} 1$, muito baixo, sem que entretanto o aumento de $\mathrm{P} 3$ e P2 se acentuasse com o grau de desvio. Uma forma particular, até certo ponto característica, é uma tendência para um $\mathrm{P} 1$ isoelétrico e difásico, assim como, principalmente, a sua negatividade.

Em resumo, portanto, o $P$ pulmonar se caracterisa por um aumento, sem entalhes, da onda em DII e DIII e por um achatamento ou mesmo um difasismo dela em. DI.

\section{3. ${ }^{\mathrm{a}}$ Parte}

\section{EXPOSIÇÃO DE NOSSOS DADOS E CONCLUSÕES}

Diante desses estudos sobresai a possibilidade de se distinguir, no eletrocardiograma, pelas alterações da onda $\mathrm{P}$, os quadros mitrais dos pulmonares. Dado o interesse desses fatos, procuramos, em 24 casos, verificar a procedência de'tais anomalias. Naturalmente, não pudemos positivar, à necropsia, se as alterações encontradas correspondiam à hipertrofia de uma ou de ambas as auriculas. De momento, não é isso que nos interessa. Desejamos, apenas, verificar se, com os achados eletrocardiográficos, podemos, de fato, distinguir ou, pelo menos suspeitar as lesões mitrais das pulmonares, isto é, se na maioria dos casos, encontramos, naquelas situações, quadros mais ou menos típicos. 


\section{NOSSO MATERIAL}

As nossas conclusões resultaram na analise de 24 casos, dos quais 12 de estenose mitral, extraidos dos arquivos da $2 .^{\mathrm{a}} \mathrm{M}$. H. e outros 12, de doença cárdio-pulmonar. Destes, 4 foram retirados dos ar-

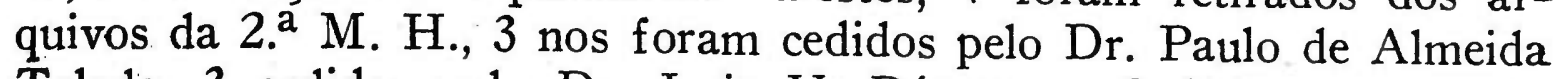
Toledo, 3 cedidos pelo Dr. Luiz V Décourt e 2 últimos, por nós observados na 2. a $^{\mathrm{a}}$ Enfermaria de Homens.

Nos quadros anexos, reproduzimos os dados sobre os quais se fundamentam as nossás conclusões. Além desses, analisámos em todos os casos a idade, o biotipo, a etiologia, o eixo elétrico, à duração do espaço PQ e o resultado do exame radiológico.

Vejamos, portanto, quais as condições que pudemos tirar.

P. PULMONAR

\begin{tabular}{|c|c|c|c|c|c|c|c|c|}
\hline \multirow{2}{*}{ CASOS } & \multicolumn{3}{|c|}{ Duração (1) } & \multicolumn{3}{|c|}{ Amplitude (2) } & \multirow{2}{*}{$\begin{array}{l}\text { Eixo } \\
\text { de p }\end{array}$} & \multirow{2}{*}{ Entalhe } \\
\hline & p1 & p2 & p3 & p1 & $\mathrm{p} 2$ & p3 & & \\
\hline $\begin{array}{lr}\text { CASO } & 1 \\
\text { CASO } & \mathbf{2} \\
\text { CASO } & \mathbf{3} \\
\text { CASO } & 4 \\
\text { CASO } & \mathbf{5} \\
\text { CASO } & \mathbf{6} \\
\text { CASO } & 7 \\
\text { CASO } & 8 \\
\text { CASO } & \mathbf{9} \\
\text { CASO } & 10 \\
\text { CASO } & 11 \\
\text { CASO } & 12\end{array}$ & $\begin{array}{l}0 " \\
0 \\
0,14 \\
0,08 \\
0,10 \\
0,08 \\
0,04 \\
0 \\
0 \\
0,10 \\
0,04 \\
0\end{array}$ & $\begin{array}{l}0,08 \\
0,10 \\
0,14 \\
0,10 \\
0,12 \\
0,10 \\
0,10 \\
0,10 \\
0,04 \\
0,08 \\
0,10 \\
0,10\end{array}$ & $\begin{array}{c}0,08 \\
0,08 \\
0,08 \\
0 \\
0,08 \\
0,08 \\
0,08 \\
0,10 \\
0,08 \\
0,08 \\
0,10 \\
0,08\end{array}$ & $\begin{array}{c}0 \\
0 \\
0,5 \\
2 \\
1 \\
0,5 \\
0,5 \\
0 \\
0 \\
1 \\
1 \\
0,5\end{array}$ & $\begin{array}{c}3,5 \\
3,4 \\
1 \\
2 \\
2 \\
3 \\
2,5 \\
2 \\
0,5 \\
3 \\
2,5 \\
2,5\end{array}$ & $\begin{array}{c}3,5 \\
3,4 \\
0,5 \\
0 \\
1 \\
2,5 \\
2 \\
2 \\
0,5 \\
2 \\
1,5 \\
2\end{array}$ & $\begin{array}{l} \pm 90 \\
\pm \quad 90 \\
\pm \quad 60 \\
\pm \quad 30 \\
\pm \quad 20 \\
\pm \quad 85 \\
\pm 95 \\
\pm 90 \\
\pm 90 \\
\pm 125 \\
\pm \quad 51 \\
\pm \quad 70\end{array}$ & p1 \\
\hline
\end{tabular}

(1) Todos os dados de duração se referem a frações de sẻgundo.

(2) As amplitudes são medidas em milímetros.




P MITRAL

\begin{tabular}{|c|c|c|c|c|c|c|c|c|}
\hline \multirow{2}{*}{ CASUS } & \multicolumn{3}{|c|}{ Duração (1) } & \multicolumn{3}{|c|}{ Amplitude (2) } & \multirow{2}{*}{$\begin{array}{l}\text { Eixo } \\
\text { de p }\end{array}$} & \multirow{2}{*}{ Entalhe } \\
\hline & $\mathrm{p} 1$ & $\mathrm{p} 2$ & p3 & $\mathrm{p} 1$ & $\mathrm{p} 2$ & p3 & & \\
\hline CASO 1 & 0,08 & 0,12 & 0,08 & 0,5 & 2,5 & 2 & \pm 65 & \\
\hline CASO 2 & 0,06 & 0,12 & 0,12 & 1 & 2 & 1 & \pm 60 & p2-p3 \\
\hline CASO & 0,12 & 0,12 & 0,03 & 2,5 & 2,5 & 0 & \pm 38 & \\
\hline CASO 4 & 0,08 & 0,12 & 0,08 & 1,5 & 3 & 1,5 & \pm 60 & p2-p3 \\
\hline CASO & 0,12 & 0,12 & 0,08 & 0,5 & 2 & 1,5 & \pm 50 & $\mathrm{p} 3^{\mathrm{r}}$ \\
\hline CASO & 0,12 & 0,12 & 0,10 & 4,5 & 2,5 & -2 & $\pm(-50)$ & p1-p2 \\
\hline CASO & 0,08 & 0,06 & 0,04 & 2,5 & 1,5 & 1 & $\mp(-25)$ & p1 \\
\hline CASO & 0,12 & 0,06 & 5,08 & 2,5 & 2 & $-0,5$ & $\pm(-10)$ & p1-p2-p3 \\
\hline CASO 9 & 0,06 & 0,08 & 0,04 & 1 & 2,5 & 15 & \pm 50 & \\
\hline CASO 10 & 0,12 & 0,08 & 0,06 & 1,5 & 1 & $-0,5$ & \pm 10 & $\mathrm{pl}$ \\
\hline CASO 11 & 0,06 & 0,08 & 0,08 & 0,5 & 1,5 & 1 & \pm 50 & p2-p3 \\
\hline CASO 12 & 0,08 & 0,10 & 0,08 & 0,5 & & 1,5 & \pm 60 & p2-p3 \\
\hline
\end{tabular}

(1) Todos os dados de duração se referem a frações de segundo.

(2) As amplitudes são medidas em mílimetros.

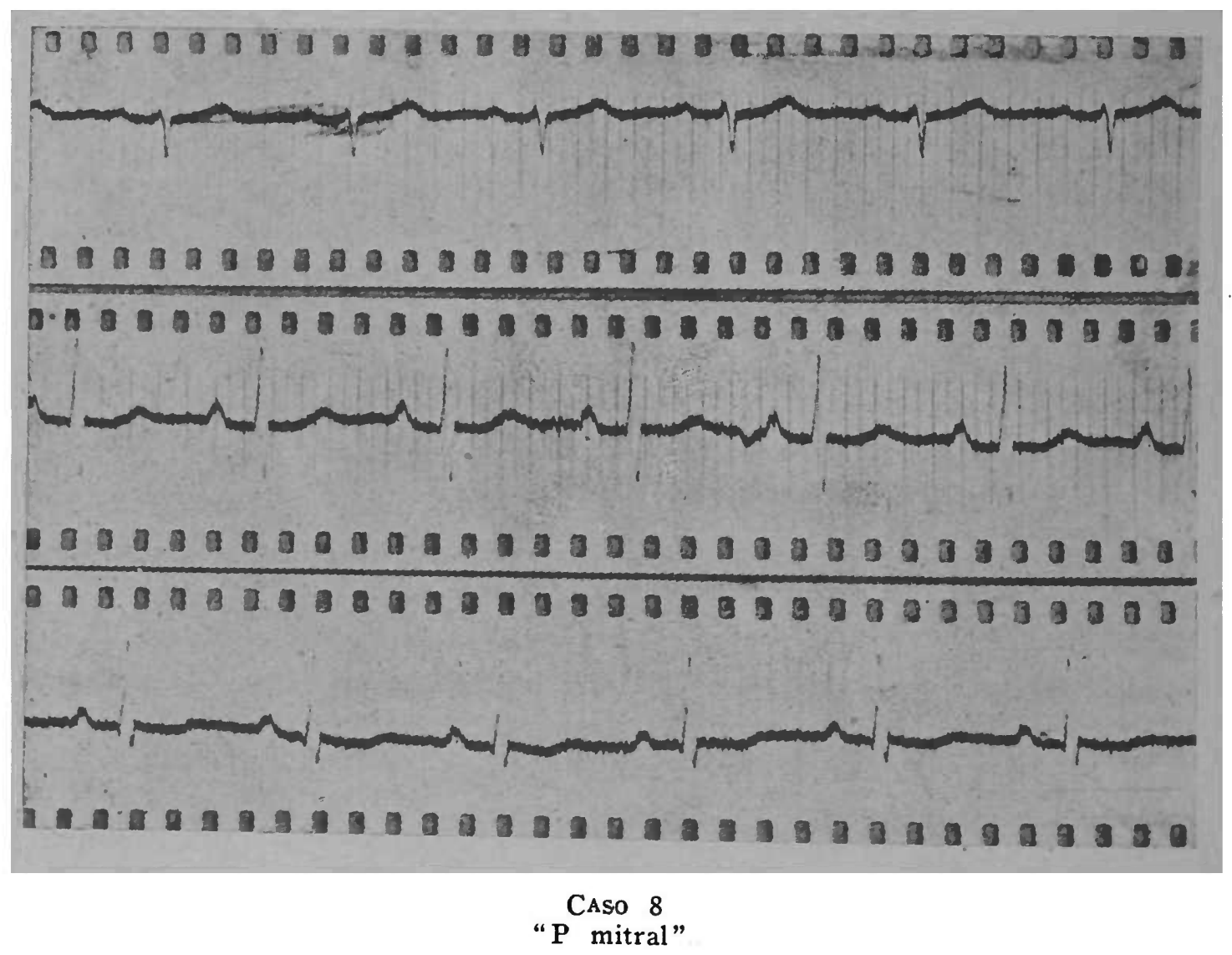

Análise da duração da onda $P$ : Apenas em 2 casos de "cor pulmonale" encontramos a onda $\mathrm{P}$ com a duração aumentada numa derivação (caso 5) ou em duas derivações (caso 3 ).

Em 8 mitrais observamos alargamento de $\mathrm{P}$ acima de 0,11 (considerado por Ashman e Hull como limite máximo da normalidade) em uma ou mais derivações. Num único caso, encontramos a onda.P alargada na $3 .^{\text {a }}$ derivação (caso 2 ), portanto, quasi todos os alargamentos foram verificados em D1 ou D2. 
Análise da amplitude da onda $P$ : Em 8 dos 12 casos de "cor pulmonale" verificamos um $\mathrm{P}$ chato ou isoelétrico em $\mathrm{D} 1$.

Em 10 casos P2 mostrou-se aumentado, atingindo em dois casos (caso 1 e caso 2) respectivamente valores de $3^{\mathrm{mm}}, 5$ e $3^{\mathrm{mm}}, 4$.

Em 9 casos P3 mostrou-se com amplitude acima do normal; nos casos 1 e 2 esta apresentou os valores de $3^{\mathrm{mm}}, 5$ e $3^{\mathrm{mm}}, 4$.

Analisando em conjunto, a onda $\mathrm{P}$ nas 3 derivações, verificamos em 6 casos, ao lado de uma diminuição na amplitude de P1, um aumento em $\mathrm{P} 2$ e' $\mathrm{P} 3$. Em 3 cåsos notamos aumento na amplitude de P2 e P3 tendo, entretanto, se conservado normal P1.

Em 8 ou 12 casos de mitrais encontramos P1 mais amplo que o normal, sendo que no caso 6 a amplitude de P1 mediu $4^{\mathrm{mm}}, 5$.

Em 9 casos encontramos um P2 mais amplo que normalmente.

Em 5 casos encontramos um P3 isoelétrico (um caso) ou negativo (4 casos).

Apenas, em 4 dos 12 casos examinados verificamos ao lado de um $\mathrm{P} 3$ chato ou negativo um $\mathrm{P} 1$ e $\mathrm{P} 2$ amplos.

Análise do eixo de $P$ : Em 8 casos de cárdio-pulmonares encontramos o eixo de $\mathrm{P}$ desviado nitidamente para a direita (com valores acima de +60$)$. .

Em todos os casos de $\mathrm{P}$ pulmonar a onda $\mathrm{P}$ se apresentou com um eixo tendo um valor superior a +60 .

Em 7 casos de mitrais observamos o eixo de $\mathrm{P}$ com valorés abaixo de +60 , sendo que 3 desses apresentaram valor negativo.

Análise da forma da onda $P$ : Num único caso onde o diagnóstico clínico fora de "cor pulmonale" encontramos um entalhe (caso 4) sendo interessante notar que, ai encontramos tambem uma onda $P$ de tipo mitral.

Em 9 casos de mitrais encontramos o acidente $\mathrm{P}$ com alterações na forma, sendo que em 6 desses, o entalhe foi notado em mais de uma derivação.

\section{CONCLUSÓES}

1) $\mathrm{O}$ alargamento de $\mathrm{P}$ é mais comum no tipo mitral, onde, frequentemente, encontramos a associação de estenose mitral com a insuficiência mitral.

2) Além da quase absoluta inalterabilidade na forma do $\mathrm{P}$ pulmonar consideramos de real valor para o diagnóstico o aumento de P2 e P3 e a diminuição na amplitude de P1.

3) Consideramos mais valiosas, para o diagnóstico de hipertrofia da aurícula esquerda, as alterações na forma da onda $P$, do que os aumentos na amplitude de P1 e P2 com um P3 relativamente chato.

4) São importantes os desvios para a direita do eixo de $P$ nos cárdio pulmonares e para a esquerda nos mitrais.

5) Nos mitrais a presença de entalhe na onda $P$ foi observada com uma frequência digna de nota. 


\section{BIBLIOGRAFIA}

1 - AsHman, R. e Hull, E. - "Essentials of Eletrocardiography" New York, The Macillan Company, pgs. 28-38,-1937.

2 - Berliner, K. e Master, A. M. - "Mitral Stenosis - A correlation os electrocardiographic and pathologic observations" - Arch. Int. Med., 61; 39, 1938.

3 - Best e TAYLOR - "The physiological basis of medical practice" - The William \& Wilkins Company - Baltimore, pg. 293, 1940.

4 - Pardee, H. E. B. - "Clinical Aspects of the Eletrocardiogram". New York, Paul B. Hoeber, Inc., pg. 109-112; 35-37; 77-80. 1941.

5 - Ramos, J. — "Lições de eletroc̀ardiologia clínica" - Companhia Editọra Nacional - pgs. 107-110. 1935.

6 - Schlomka, G. Klein, W. - Beiträge zur klinischen Elektrokardiographie. IX - Zur Bewertung der $P$ Zacke bei rechtstypischen Elektrokardiogrammen. Zeit. klin. Mediz. 133, 648. 1938.

7 - Toledo, P. A. - "Eletroradiologia clínica do coração" - Companhia Editora Nacional, pgs. 329-332. 1940.

8 - Toledo, P. A. - As hipertensões da pequena circuação. Arq. Cir. Clín. e Exp. Numero especial comemorativo do jubileu professoral do prof. dr. Antonio de Almeida Prado., Pg. 835-890. Junho-Agosto, 1941.

9 - Winternitz, M. - Zur Pathologie des menschlichen Vorhofelektrokardiogramms. Med. Klinik, 31; 1575.1935.

\section{Preparad os Farmacêt i cos}

Temos a venda Marcas e Formulas licenciadas e incumbimo-nos de sua compra, venda, ou registro. LICENCIAMOS FORMULAS, PODENDO SER EXAMINADAS POR NOSSO TECNICO FARMACEUTICO OU FORNECER FORMULAS. Legalizamos Laboratorios Farmaceuticos, fazemos quaisquer contratos, de instalação, de exploração, de propaganda, de fabricação.

C O N S U T E M-N OS SEM COMPR OMIS O

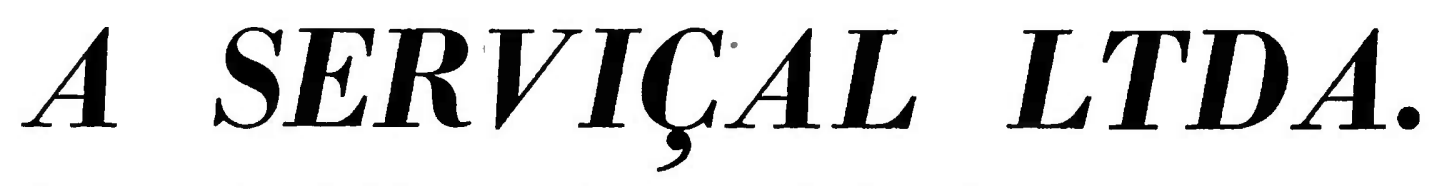

\section{agencias Reunidas Rio de JANEIRo E S. PaUlo}

\section{Diretor Geral: ROMEU RODRIGUES}

Marcas Parentes e Licenças de Preparados Fármacêulicos Comestiveis Bebidas Elc.

RIO DE J A N EIRO

Rua da Quitanda, 7-Sob.

(Perto da Rua S. José)

Caixa postal, 3384

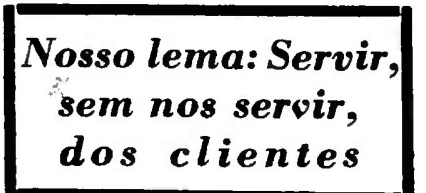

$\begin{array}{lllllllll}\mathbf{S} & \mathbf{A} & \mathbf{O} & \mathbf{P} & \mathbf{A} & \mathbf{U} & \mathbf{L} & \mathbf{O}\end{array}$

Rua Direita, 64 $\cdot 3 . .^{\circ}$ And.

CAIXAS POSTAIS

3631 e 1421 